\title{
Characterizing Clinical Outcomes and Factors Associated with Conduction Gaps in VISITAG SURPOINT-Guided Catheter
} Ablation for Atrial Fibrillation

\author{
Koichi Inoue ${ }^{1}$, Nobuaki Tanaka ${ }^{1}$, Yusuke Ikada ${ }^{1}$, Akinobu Mizutani ${ }^{1}$, Kazuhiko \\ Yamamoto $^{1}$, Hana Matsushima ${ }^{1}$, Shinichi Harada ${ }^{1}$, Masato Okada ${ }^{1}$, Katsuomi Iwakura ${ }^{1}$, \\ and Kenshi Fujii ${ }^{1}$ \\ ${ }^{1}$ Sakurabashi Watanabe Hospital
}

October 21, 2020

\begin{abstract}
Background: Few studies have explored the link between VISITAG SURPOINT (VS) parameters and the inter-tag distance (ITD) on pulmonary vein isolation (PVI) in catheter ablation of atrial fibrillation (AF). Objective: To examine the clinical outcomes and factors contributing to residual conduction and reconnection gaps during PVI. Methods and Results: A retrospective review of consecutive patients $(\mathrm{N}=100)$ with paroxysmal $(\mathrm{n}=32)$ or persistent AF $(\mathrm{n}=68)$ undergoing VS-guided ablation between 09/2018-08/2019 was performed. All procedures were performed by 2 operators. Target VS values were 425 (anterior), 375 (posterior), and 325 (in proximity of the esophagus). Target ITD was $4 \mathrm{~mm}$. Acute PVI was achieved in $100 \%$ of cases, however 13 residual gaps in 12 patients were observed after the first encirclement (first pass isolation: 88\%). Ten gaps due to spontaneous PV reconnections (PVR) were found in 9 patients (9\%). Tags associated with these 23 gaps had similar median VS (gap-related vs. non-gap: 429 vs. $410, \mathrm{p}=0.4545)$ and power ( 36 vs. $36 \mathrm{~W}, \mathrm{p}=0.4843)$, higher contact force $(13.8 \mathrm{vs}$. $11.0 \mathrm{~g}$, $\mathrm{p}=0.0061)$, and larger ITD (5.3 vs. $3.7 \mathrm{~mm}, \mathrm{p}<0.001)$ when compared to the remaining tags. At median follow-up of 462 days $(\mathrm{N}=99$ patients), freedom from any atrial arrhythmia was $84.8 \%$. Eight patients received repeat ablation $(8.1 \%)$ and of these, $6(75 \%)$ were free from PVR. No major adverse events were noted. Conclusion: Favorable rates of first pass isolation, acute PVR, and long-term procedure success were achieved using lower VS values than in previous reports. Under these settings, larger ITDs are associated with residual conduction and reconnections.
\end{abstract}

\section{Introduction}

Atrial fibrillation (AF) is the most common form of cardiac arrhythmia. ${ }^{1}$ Although radiofrequency (RF) catheter ablation is an established and recommended treatment option for paroxysmal AF (PAF) ${ }^{2}$ recovery of ablated tissue and reconnection of at least one pulmonary vein (PV) has been reported in up to $70 \%$ of AF patients following pulmonary vein isolation (PVI). ${ }^{2,3}$ In fact, pulmonary vein reconnection (PVR) has been identified as a major driving factor of AF recurrence, ${ }^{4-6}$ which may result in repeat ablations, increased medical costs, ${ }^{7}$ and higher risk of complications. ${ }^{8}$ Creating durable lesions during PVI is therefore critical to prevent PVR and subsequent recurrence of arrythmia. ${ }^{2,9,10}$

The adoption of real-time contact force $(\mathrm{CF})$ monitoring in $\mathrm{RF}$ catheter ablation has optimized acute and long-term procedural success by providing operators with information on catheter stability and tissuecatheter contact. ${ }^{11-13}$ However, factors other than CF have been shown to affect lesion formation (e.g., energy delivery, time, and RF power). ${ }^{14}$ VISITAG SURPOINT (VS; previously Ablation Index) is a module available in the CARTO3 System that allows automated annotation and tagging of RF ablation applications. In clinical studies, VS-guided ablation has demonstrated high durability in PVI and arrhythmia-free survival up to 12-14 months after an index ablation procedure. ${ }^{15-20}$ 
Although benchmark studies have reported the safety, efficacy, clinical applicability, and procedural efficiency outcomes associated with the use of VS, ${ }^{15-22}$ these studies have recommended definitive VS thresholds while providing relatively general guidance on the inter-tag distance (ITD), which is expected to influence lesion contiguity and PVI durability. A recent randomized controlled study reported that lower target ITD was associated with better acute procedural outcomes, suggesting that smaller ITDs may allow for less extensive ablation per lesion. ${ }^{28}$ Therefore, the efficacy of PVI with lower target ITD and VS values should be validated. The objective of this single-center, retrospective, observational study was to examine the clinical outcomes of catheter ablation with lower target ITDs and less intensive ablation at each lesion, along with factors contributing to residual conduction and reconnection gaps, during catheter ablation for AF.

\section{Methods}

\section{Study Population}

From September 1, 2018 to August 1, 2019, 100 consecutive adult patients ([?]18 years of age) with drug resistant symptomatic AF (PAF or persistent [PsAF]) who underwent VS-guided circumferential ipsilateral PVI by two operators at the Sakurabashi Watanabe Hospital in Osaka, Japan were enrolled. Exclusion criteria included subjects with long-standing PsAF at diagnosis, prior ablation for AF, or those with deterioration of renal function (i.e., creatine clearance below $30 \mathrm{~mL} / \mathrm{min} / 1.73 \mathrm{~m}^{2}$ ). Those who received extensive empiric ablation beyond PVI were also excluded. Patients were maintained on oral anti-coagulation for at least 1 month prior to ablation. Warfarin was interrupted 1 day before, and resumed on the evening of the procedure. Anti-arrhythmic drugs (AADs) were stopped at least 5 half-lives before ablation. Dabigatran, rivaroxaban, and apixaban were omitted only on the morning of the procedure. ${ }^{29}$ The study protocol was approved by our institutional ethics committee prior to data collection. Individual patient informed consent was waived and the opt-out method for patient recruitment was used due to the retrospective study design.

\section{Ablation Procedure}

All patients underwent 3D reconstruction of the left atrium (LA) and PV prior to AF ablation via multidetector computed tomography (CT) (Brilliance CT 64; Philips Medical Systems, Cleveland, OH, USA). For cardiac catheterization, two decapolar catheters were placed at the coronary sinus and in the superior vena cava and right atrium, respectively. An intracardiac echocardiography (ICE) catheter was inserted into the right atrium via the femoral vein (Soundstar Catheter; Biosense Webster, Inc., Irvine, CA, USA). Three-dimensional visualization of the LA was achieved using the CARTO3 System with the CartoSound, CartoMerge, ${ }^{30}$ and VISITAG SURPOINT modules (Biosense Webster, Inc., Irvine, CA, USA). A single trans-septal puncture was performed after introduction of two long sheaths (Daig SL0; St. Jude Medical, St. Paul, MN, USA). Subjects underwent conscious sedation with a combination of bolus thiamylal sodium, pentazocine, and continuous dexmedetomidine hydrochloride. Bi-level positive airway pressure and an oropharyngeal airway were employed to maintain stable respiration.

Settings for VISITAG were as follows: 1) catheter stability range of motion $<1.5 \mathrm{~mm}, 2$ ) catheter stability duration $>5$ seconds, and 3) $\mathrm{CF}>5 \mathrm{~g}$, time $>25 \%$. Tag size was set to $3 \mathrm{~mm}$ in diameter. We targeted VS values of 425 for the anterior wall, 375 for the posterior wall, and 325 in the proximity of the esophagus, attempting to meet or exceed these thresholds at each lesion. The target ITD was $<4.0 \mathrm{~mm}$ following standard ablation protocols at our institution.

Procedures were performed using a ThermoCool SmartTouch SF ablation catheter and a Lasso-Nav circular mapping catheter (Biosense Webster, Inc., Irvine, CA, USA). Ipsilateral circumferential PVI was conducted in sinus rhythm except in PsAF patients refractory to electrical cardioversion. Radiofrequency energy was delivered via a point-by-point approach guided by VS. We aborted RF application near the esophagus if the temperature crossed $40^{\circ} \mathrm{C}$, as measured with a SensiTherm probe (St. Jude Medical, St. Paul, MN, USA). Isolation (entrance and exit block) was confirmed after a minimum 20 minute waiting period. If residual potential persisted after first-pass circumferential PV ablation or reappeared during the procedure, gaps were mapped and ablated. Upon elimination of the PV potential we performed bonus energy delivery on both sides of the successful points. Intravenous adenosine triphosphate (ATP) challenge to trigger dormant 
conduction was not performed based on our previous study. ${ }^{31}$ Atrial flutter (AFL) or atrial tachycardia (AT) induced by burst pacing was managed via linear ablation of the cavotricuspid isthmus (CTI), roof lines, mitral valve isthmus, and focal atrium tachycardia at the operator's discretion.

Demographic and Procedure Data

\section{Patient characteristics}

Data consisting of age, sex, height, and body weight were collected at baseline. Additional variables included medication use (anti-coagulation, beta-blocker, and AADs), prior cardiovascular events (i.e., transient ischemic attack [TIA], myocardial infarction [MI], and stroke), comorbidities, and $\mathrm{CHA}_{2} \mathrm{DS}_{2}$-VASc scores. Left atrial volume (LAV), left atrium diameter (LAD), and left ventricular ejection fraction (LVEF) were recorded based on cardiac $\mathrm{CT}$ records.

\section{Procedure and ablation lesion data}

Date of admission and discharge, ablation performed (i.e., PVI, CTI, linear, and others), electrophysiology (EP) lab time (i.e., from patient entrance to exit), ablation time, RF duration, fluoroscopy time, and fluoroscopy dose were analyzed to describe the index procedure characteristics.

For VS data, we divided conduction gaps into 'residual gaps' and 'reconnection gaps' based on their timing and characteristics. Pulmonary vein electrical conduction remaining after an initial anatomical burn was referred to as a 'residual gap'. Acute reconnections (whether located at residual gaps or elsewhere) detected during the rest of the procedure were named as a 'reconnection gaps'. Where additional RF energy was applied for touch-up, 'success tags' were assigned to the last tag where local PV potential was confirmed to have disappeared. The two adjacent tags associated with the success tags were termed 'gap-related tags'. The remaining tags from the first encirclement were referred to as 'non-gap tags'. These definitions are shown inFigure 1. Each PV was divided into 6 segments (i.e., roof, superior anterior, inferior anterior, superior posterior, inferior posterior, and inferior) for subsequent analyses. Including 2 additional areas near the esophagus and otherwise unclassified regions, a total of 14 segments were assigned by CARTO operators (Figure 2).

\section{Characterizing Residual and Reconnection Gaps}

The number of tags and their individual parameters (i.e., RF duration, CF, power, VS, ITD, and impedance drop) were analyzed, especially in relation to residual gaps and reconnection sites. A proprietary algorithm (coded in MATLAB version 9.2, The MathWorks Inc., Natick, MA, USA) was employed to visualize all tags in three dimensions, to identify lesions associated with the first-pass wide antral circumferential ablation line, and to calculate ITDs.

\section{Long-Term Follow-up}

All patients were hospitalized under continuous rhythm monitoring for 3 days after the ablation procedure. We directed patients to check their heart rate and rhythm at least 3 times per day during post-ablation followup, and to visit the outpatient clinic if they experienced a relapse. All patients were scheduled for routine follow-up visits to the outpatient clinic at 1, 2, 3, 6, 9, and 12 months. Electrocardiograms were obtained at each visit. A Holter electrocardiogram was performed at the 6 month visit. Additional monitoring was performed on the basis of symptoms. A 3 month blanking period was used. Freedom from atrial arrhythmias was defined as the absence of AF, AFL, or AT lasting > 30 seconds either on or off AADs.

Statistical Analysis

Frequency counts and proportions are provided for binary and categorical data. Continuous variables were reported either as mean \pm standard deviation (SD) or median (interquartile range $[\mathrm{IQR}]$ ) depending on Normality testing. For continuous variables, comparisons between independent groups were performed using a two sample Student's t test if normally-distributed, or the Wilcoxon rank sum test (Mann-Whitney U test) 
otherwise. The Chi-Square test was used for binary and categorical variables. A p-value $<0.05$ was deemed to be statistically significant.

Binomial and multiple regression analyses were conducted to examine covariates that may affect outcomes. Covariates were selected based on clinical knowledge and included the patient sex, AF type, AAD use at baseline, exact $\mathrm{CHA}_{2} \mathrm{DS}_{2}$-VASc score, $\mathrm{CHA}_{2} \mathrm{DS}_{2}$-VASc score [?] 2 vs. 0 or 1, hypertension, diabetes, vascular disease, and PVI only vs. additional ablation strategies. All statistical analyses were performed using $\mathrm{R}$ Statistical Software (version 3.6.3, The R Development Core Team, Vienna, Austria).

\section{Results}

Patient Population

The study population comprised 100 patients with a primary diagnosis of PAF $(\mathrm{n}=32)$ or PsAF $(\mathrm{n}=68)$. Demographic and baseline data are summarized in Table 1 . Median age was 67 (IQR: 59, 74) years. A majority were male $(76 \%)$. The most common comorbidities among all patients were hypertension $(59 \%)$ and diabetes mellitus (22\%). Most patients (64\%) had a $\mathrm{CHA}_{2} \mathrm{DS}_{2}$-VASc score above 2.

Procedural Characteristics

In addition to PVI, $31 \%$ and $10 \%$ of patients received CTI and linear ablation, respectively, due to clinical ATs or arrhythmias induced by burst pacing; $4 \%$ were ablated at superior vena cava triggers initiating AF $(\mathrm{n}=2)$ and non-PV foci for ATs $(\mathrm{n}=1)$, and for premature atrial contractions $(\mathrm{n}=1)$. Median EP lab time was $145.3(130.9,163.1)$ minutes. The median fluoroscopy time and dose were $7.2(4.4,9.7)$ minutes and $17.5(8.8,42.0)$ mGy. Total RF application time was $27.3(23.8,31.0)$ minutes.

\section{Acute Clinical Outcomes}

Data on acute clinical outcomes are presented in Figure 3. First pass isolation (FPI) was accomplished in both ipsilateral PVs for 88 patients (88\%), with 13 gaps remaining after the first encirclement. Nine patients (9\%) experienced spontaneous PVR due to 10 reconduction gaps. Since 2 patients had both residual and reconnection gaps (Supplemental Table 1 ), 81 were free from any PVI gaps prior to touch-up. Acute procedural success was achieved in $100 \%$ of patients. No major adverse events (AEs) occurred in the periprocedural period. One right femoral arteriovenous fistula and 4 acute gastric dilatations were detected. None required surgery or intervention, and all complications resolved without permanent clinical sequelae. Binomial and multiple regression analyses showed no significance of co-variates on any acute clinical outcomes (FPI, PVR, or acute success).

VS Parameter Analysis

A total of 7,399 tags were recorded during the procedures. VS parameters stratified by segment are presented in Table 2 . Although a substantial number of tags did not strictly satisfy their segment-specific VS threshold values (anterior: $26.9 \%$, posterior: $37.1 \%$, and near esophagus: $64.3 \%$ ), deviations remained fairly minimal: $434(419,441)$ in the anterior (target $=425), 384(337,403)$ in the posterior (target $=375)$, and $303(269$, 332 ) near the esophagus (target $=325$ ). ITDs were consistently below $4.0 \mathrm{~mm}$ in all segments except near the esophagus.

\section{Residual Gaps}

Thirteen residual gaps occurred in 12 patients and were successfully isolated with additional RF applications. These were most commonly observed in the anterior $(7 / 13,53.8 \%)$ as opposed to the posterior $(5 / 13,38.5 \%)$ and roof $(1 / 13,7.7 \%)$ segments (Figure 2 ).Figures $\mathbf{4 A}$ and $\mathbf{4 B}$ depict the box plots for VS values and ITDs based on tag classifications (i.e., non-gap, gap-related, and success). As shown in panel A, there was no difference in the median VS values between gap-related and non-gap tags $(424$ [352, 439] vs. 410 [361, 435], $\mathrm{p}=0.8262)$. VISITAG SURPOINT values $(437+-38)$ beyond the anterior segment target were applied to achieve successful elimination of residual gaps. On ITD, ablation tags contributing to residual gaps were found to be statistically longer $(5.3[5.0,6.1]$ vs. $3.7[2.8,4.7] \mathrm{mm}, \mathrm{p}<0.001)$. 


\section{Reconnection Gaps}

Ten spontaneous reconnections occurred in 9 patients and were terminated with additional RF applications. These were most commonly observed in the anterior $(6 / 10,60.0 \%)$ versus the posterior $(2 / 10,20.0 \%)$, roof $(1 / 10,10.0 \%)$, or near the esophagus $(1 / 10,10.0 \%)$ segments.Figures $\mathbf{4 C}$ and $\mathbf{4 D}$ depict the box plot for VS values and ITDs based on tag classifications (i.e., non-gap, gap-related, and success). Similar to the residual gap analysis, there was no difference between median VS values for reconnection versus non-reconnection tags $(430[389,434]$ vs. $410[361,435], \mathrm{p}=0.3842)$. A high VS value $(436+-41)$ applied to reconduction sites resulted in successful elimination of these gaps. On ITD, ablation tags contributing to reconnections were also statistically longer $(4.6[4.1,7.1]$ vs. $3.7[2.8,4.7] \mathrm{mm}, \mathrm{p}=0.0141)$.

Features of Gap-Related Tags and Non-Gap Tags

A total of 23 gaps (13 residual gaps and 10 reconnection gaps) were observed in 19 patients (Supplemental Table 1 ), and therefore 46 gap-related, 23 success, and 7,330 non-gap tags were identified in this study. Gaps were found more commonly in the right versus the left PV (residual gaps: right $=8$, left $=5$; reconnection gaps: right $=7$, left $=2$ ). One reconnection gap was observed near the esophagus. Four gaps were located in the carina, 1 of which was a residual gap and 3 of which were reconnection gaps. Table 3 illustrates that the median VS value of non-gap tags were similar to that of gap-related tags (410 [361, 435] vs. 429 [384, $435], \mathrm{p}=0.4545)$. On the other hand, ITD was significantly longer among gap-related tags (median: 5.3 $[4.3,6.3]$ vs. $3.7[2.8,4.7] \mathrm{mm}, \mathrm{p}<0.001)$.

\section{Long-Term Clinical Outcomes}

We obtained follow-up data for 99 patients at a median follow-up duration of 462 +- 110 days. Eighty-four $(84.8 \%)$ were free from any atrial tachyarrhythmias based on standard-of-care monitoring. At 1-year, the Kaplan-Meier survival from AT/AF was 87.2\% (95\% Confidence Interval: $80.4-94.0 \%$ ) (Figure 5 ). There was no significant difference between patients on the recurrence-free rate based on the presence or absence of gaps (with vs. without: $94.7 \%$ vs. $82.5 \%, \mathrm{p}=0.2901$ ). Eight patients $(8.1 \%)$ received re-ablation at a median $239+-141$ days after the index procedure. Amongst these, $75.0 \%$ (6/8) of patients and $84.4 \%$ $(27 / 32)$ of PVs remained fully isolated. No procedure-related AEs were reported in any patients.

\section{Discussion}

This study demonstrates safe and effective AF ablation at lower target ITD and VS values than commonly reported, with a high FPI (88\%), low acute PVR (9\%), durable lesions (84\% PVs isolated), and high long-term procedural success (85\%). Our results suggest that beyond VS thresholds, ITD is an important determinant of procedural success due to its impact on lesion contiguity. Durable PVI is critical to prevent late tissue recovery and arrhythmia recurrence; therefore, these findings help inform best practice in a realworld clinical setting.

The safety, efficacy, clinical applicability, and procedural efficiency outcomes associated with VS-guided AF ablation have previously been reported in benchmark studies. ${ }^{15-22}$ Specifically, the CLOSE protocol described low rates of PVR and promising long-term success using an ITD [?] $6 \mathrm{~mm}$ and target VS of 550/400 at the anterior/posterior walls. ${ }^{20}$ VISTAX was the first multi-center study exploring reproducibility of the CLOSE protocol for 340 patients with PAF, and reported an $82.4 \%$ FPI rate and 12-month freedom from AT/AF of $89.4 \%$ across 17 sites. ${ }^{15}$ Our protocol adopted a target ITD of $4 \mathrm{~mm}$ and target VS of $425 / 375$ at the anterior/posterior walls, which were lower than those in the CLOSE protocol. A recurrence-free rate of $84.8 \%$ was achieved during median follow-up of 462 days. This value appears slightly low compared with reports of up to $94 \%$ single-procedure freedom from arrhythmias ${ }^{18}$ in PAF ablation after 1 year. Nevertheless, our findings may be reasonable because two-thirds of the study population consisted of persistent AF patients, where outcomes are generally worse than in PAF. ${ }^{27}$ In spite of lower target values of ITD and VS, our results are consistent with the VISTAX study, ${ }^{15}$ which indicates that our protocol is also sufficient to obtain reasonable clinical outcomes.

Relatively few studies have described the use of lower target VS values. ${ }^{25,26}$ In the OPTIMUM study, Lee 
et al. evaluated the optimal VS values for avoiding acute PVR and examined the feasibility and efficacy of these targets among a Korean population. ${ }^{23}$ Authors found that VS values [?] 450 for the anterior/roof segments, [?] 350 for the posterior/inferior/carina segments, and an ITD of [?] $4 \mathrm{~mm}$ decreased acute PVR compared to conventional PVI ablation without the use of VS. Okamatsu et al. investigated the role of power on VS-guided ablation using a target VS of 400 for the anterior, 360 for the posterior, and 260 for the oesophagus. ${ }^{24}$ Results suggested lower VS settings may be effective in creating durable lesions regardless of $\mathrm{RF}$ power.

We also investigated multiple VS parameters to elucidate the correlation between ablation settings and conduction gaps. Despite applying lower VS values, this parameter itself was not associated with residual conduction and reconnection tags. On the contrary, larger ITDs demonstrated a strong association despite being fairly close. In fact, the mean ITD for gap-related tags was $5.3 \mathrm{~mm}$, which is within the range set by the CLOSE protocol. Hoffman et al. recommended to aim for an ITD of $3.0-4.0 \mathrm{~mm}$, rather than $5.0-6.0 \mathrm{~mm}$, to optimize FPI based on results of a randomized controlled trial. ${ }^{28}$ These data highlight that "optimal" VS thresholds can vary depending on ITDs and vice versa. Closer ITDs may allow for less extensive ablation per lesion.

Unexpectedly, we found that higher average CF was associated with gaps. However, we speculate these results may not necessarily imply that high CF leads to gap formation. Statistically this factor was significant; yet clinically speaking, these differences were minimal (CF: 2.8g) with values well in the range of prior studies. Within a certain window, stability of the catheter location and $\mathrm{CF}$ are more critical than the actual CF value. To illustrate this point, the mean $\mathrm{CF}$ achieved in SMART $\mathrm{AF}^{12}$ and SMART $\mathrm{SF}^{33}$ were $17.9 \mathrm{~g}$ and $16.7 \mathrm{~g}$, respectively. The difference between these studies is similar in magnitude to our findings, but long-term outcomes did not vary markedly.

Finally, it was a little surprising that the presence or absence of gaps during the index procedure was not associated with the recurrence of AT/AF. This is possibly because all gaps were ablated carefully and intensively. In our series, $84 \%$ of $\mathrm{PVs}$ remained isolated in patients who received repeat ablation. Thus, VS-guided PVI performed under the described settings appears durable with re-ablations caused primarily by non-PV mechanisms.

\section{Limitations}

There are a few limitations to this study. First, it was retrospective, observational, and without randomization; therefore, selection bias could not be controlled. Second, during the follow-up period, patients received limited assessment of recurrence. Freedom from atrial arrhythmias might have been overestimated accordingly in case of asymptomatic AT/AF. Third, while the participants in prior benchmark studies were mainly Caucasian, ${ }^{15-22}$ all our study subjects were Asian. Because anatomic sizing and features in Asians are typically smaller, this might have impacted outcomes. Finally, results are limited to a single center with two operators. The study may not be generalizable if material changes in ablation workflows, hospital practice patterns, or VS protocols are applied elsewhere.

\section{Conclusions}

A favorable FPI, acute PVR, and long-term procedure success were achieved with VS-guided AF catheter ablation at lower target ITD and VS values than previously reported. Gap characterization showed that ITD was significantly associated with the presence of residual conduction and reconnection sites. These findings help inform best practices in a real-world clinical setting.

\section{Acknowledgments}

Medical writing services were provided by Susan Bartko-Winters, PhD, from SBW Medical Writing Inc. We also thank Yoko Ichishima for assistance with tag data extraction, Hye Jin Park, MPH, for biostatistics analysis, and Stephanie Lee, $\mathrm{PhD}$, for manuscript review.

\section{References}


1. Bai Y, Wang YL, Shantsila A, Lip GYH. The Global Burden of Atrial Fibrillation and Stroke: A Systematic Review of the Clinical Epidemiology of Atrial Fibrillation in Asia. Chest.2017;152(4):810-820.

2. Calkins H, Kuck KH, Cappato R, et al. 2012 HRS/EHRA/ECAS expert consensus statement on catheter and surgical ablation of atrial fibrillation: recommendations for patient selection, procedural techniques, patient management and follow-up, definitions, endpoints, and research trial design: a report of the Heart Rhythm Society (HRS) Task Force on Catheter and Surgical Ablation of Atrial Fibrillation. Developed in partnership with the European Heart Rhythm Association (EHRA), a registered branch of the European Society of Cardiology (ESC) and the European Cardiac Arrhythmia Society (ECAS); and in collaboration with the American College of Cardiology (ACC), American Heart Association (AHA), the Asia Pacific Heart Rhythm Society (APHRS), and the Society of Thoracic Surgeons (STS). Endorsed by the governing bodies of the American College of Cardiology Foundation, the American Heart Association, the European Cardiac Arrhythmia Society, the European Heart Rhythm Association, the Society of Thoracic Surgeons, the Asia Pacific Heart Rhythm Society, and the Heart Rhythm Society. Heart Rhythm.2012;9(4):632-696 e621.

3. Kuck KH, Brugada J, Furnkranz A, et al. Cryoballoon or Radiofrequency Ablation for Paroxysmal Atrial Fibrillation. N Engl J Med.2016;374(23):2235-2245.

4. Cappato R, Negroni S, Pecora D, et al. Prospective assessment of late conduction recurrence across radiofrequency lesions producing electrical disconnection at the pulmonary vein ostium in patients with atrial fibrillation. Circulation. 2003;108(13):1599-1604.

5. Nanthakumar K, Plumb VJ, Epstein AE, Veenhuyzen GD, Link D, Kay GN. Resumption of electrical conduction in previously isolated pulmonary veins: rationale for a different strategy? Circulation.2004;109(10):1226-1229.

6. Ouyang F, Tilz R, Chun J, et al. Long-term results of catheter ablation in paroxysmal atrial fibrillation: lessons from a 5-year follow-up. Circulation. 2010;122(23):2368-2377.

7. Mansour M, Karst E, Heist EK, et al. The Impact of First Procedure Success Rate on the Economics of Atrial Fibrillation Ablation.JACC Clin Electrophysiol. 2017;3(2):129-138.

8. Szegedi N, Szeplaki G, Herczeg S, et al. Repeat procedure is a new independent predictor of complications of atrial fibrillation ablation.Europace. 2019;21(5):732-737.

9. Cappato R, Calkins H, Chen SA, et al. Worldwide survey on the methods, efficacy, and safety of catheter ablation for human atrial fibrillation. Circulation. 2005;111(9):1100-1105.

10. Neuzil P, Reddy VY, Kautzner J, et al. Electrical reconnection after pulmonary vein isolation is contingent on contact force during initial treatment: results from the EFFICAS I study. Circ Arrhythm Electrophysiol. 2013;6(2):327-333.

11. Macle L, Frame D, Gache LM, Monir G, Pollak SJ, Boo LM. Atrial fibrillation ablation with a spring sensor-irrigated contact force-sensing catheter compared with other ablation catheters: systematic literature review and meta-analysis. BMJ Open.2019;9(6):e023775.

12. Natale A, Reddy VY, Monir G, et al. Paroxysmal AF catheter ablation with a contact force sensing catheter: results of the prospective, multicenter SMART-AF trial. J Am Coll Cardiol.2014;64(7):647-656.

13. Reddy VY, Pollak S, Lindsay BD, et al. Relationship Between Catheter Stability and 12-Month Success After Pulmonary Vein Isolation: A Subanalysis of the SMART-AF Trial. JACC Clin Electrophysiol.2016;2(6):691-699.

14. Haines DE. Determinants of Lesion Size During Radiofrequency Catheter Ablation: The Role of Electrode-Tissue Contact Pressure and Duration of Energy Delivery. J Cardiovasc Electrophysiol.1991;2(6):509515. 
15. Duytschaever M, Vigen J, De Potter T, et al. Standardized pulmonary vein isolation workflow to enclose veins with contiguous lesions: the multicentre VISTAX trial. Europace 2020 doi: 10.1093/europace/euaa157

16. Dhillon G, Ahsan S, Honarbakhsh S, et al. A multicentered evaluation of ablation at higher power guided by ablation index: Establishing ablation targets for pulmonary vein isolation. J Cardiovasc Electrophysiol. 2019;30(3):357-365.

17. Hussein A, Das M, Riva S, et al. Use of Ablation Index-Guided Ablation Results in High Rates of Durable Pulmonary Vein Isolation and Freedom From Arrhythmia in Persistent Atrial Fibrillation Patients: The PRAISE Study Results. Circ Arrhythm Electrophysiol.2018;11(9):e006576.

18. Phlips T, Taghji P, El Haddad M, et al. Improving procedural and one-year outcome after contact force-guided pulmonary vein isolation: the role of interlesion distance, ablation index, and contact force variability in the 'CLOSE'-protocol. Europace.2018;20(FI_3):f419-f427.

19. Solimene F, Schillaci V, Shopova G, et al. Safety and efficacy of atrial fibrillation ablation guided by Ablation Index module. J Interv Card Electrophysiol. 2019;54(1):9-15.

20. Taghji P, El Haddad M, Phlips T, et al. Evaluation of a Strategy Aiming to Enclose the Pulmonary Veins With Contiguous and Optimized Radiofrequency Lesions in Paroxysmal Atrial Fibrillation: A Pilot Study.JACC Clin Electrophysiol. 2018;4(1):99-108.

21. Das M, Loveday JJ, Wynn GJ, et al. Ablation index, a novel marker of ablation lesion quality: prediction of pulmonary vein reconnection at repeat electrophysiology study and regional differences in target values. Europace. 2017;19(5):775-783.

22. Munkler P, Kroger S, Liosis S, et al. Ablation Index for Catheter Ablation of Atrial Fibrillation- Clinical Applicability and Comparison With Force-Time Integral. Circ J. 2018;82(11):2722-2727.

23. Lee SR, Choi EK, Lee EJ, Choe WS, Cha MJ, Oh S. Efficacy of the optimal ablation index-targeted strategy for pulmonary vein isolation in patients with atrial fibrillation: the OPTIMUM study results. $J$ Interv Card Electrophysiol. 2019;55(2):171-181.

24. Okamatsu H, Koyama J, Sakai Y, et al. High-power application is associated with shorter procedure time and higher rate of first-pass pulmonary vein isolation in ablation index-guided atrial fibrillation ablation. $J$ Cardiovasc Electrophysiol. 2019;30(12):2751-2758.

25. Kobayashi S, Fukaya H, Oikawa J, et al. Optimal interlesion distance in ablation index-guided pulmonary vein isolation for atrial fibrillation. J Interv Card Electrophysiol. 2020; https://doi.org/10.1007/s10840-02000881-0

26. Yazaki K, Ejima K, Higuchi S, Yagishita D, Shoda M, Hagiwara N. Regional differences in the effects of ablation index and interlesion distance on acute electrical reconnections after pulmonary vein isolation. Journal of Arrhythmia. 2020;00:1-8.

27. Yamaguchi J, Takahashi Y, Yamamoto T, et al. Clinical Outcome of Pulmonary Vein Isolation Alone Ablation Strategy Using VISITAG SURPOINT in Non-Paroxysmal Atrial Fibrillation. J Cardiovasc Electrophysiol. 2020.

28. Hoffmann P, Ramirez ID, Baldenhofer G, Stangl K, Mont L et al. Randomized study defining the optimum target interlesion distance in ablation index-guided atrial fibrillation ablation. Europace 2020 doi:10.1093/europace/euaa147

29. Tanaka N, Inoue K, Tanaka K, et al. Automated Ablation Annotation Algorithm Reduces Re-conduction of Isolated Pulmonary Vein and Improves Outcome After Catheter Ablation for Atrial Fibrillation. Circ J.2017;81(11):1596-1602.

30. Inoue K. CartoMerge using SoundStar Catheter and Time Force Integral-Based Ablation for Atrial Fibrillation. International Journal of Arrhythmia. 2017;18(1):27-32. 
31. Kobori A, Shizuta S, Inoue K, et al. Adenosine triphosphate-guided pulmonary vein isolation for atrial fibrillation: the UNmasking Dormant Electrical Reconduction by Adenosine TriPhosphate (UNDER-ATP) trial.Eur Heart J. 2015;36(46):3276-3287.

32. Chinitz LA, Melby DP, Marchlinski FE, et al. Safety and efficiency of porous-tip contact-force catheter for drug-refractory symptomatic paroxysmal atrial fibrillation ablation: results from the SMART-SF trial. Europace . 2018;20(FI_3):f392-f400.

\section{Figure Legends}

\section{Figure 1: Gap and Tag Definitions}

During the ablation procedure, "success tags" (purple) were associated with lesions where additional RF energy delivery terminated dormant conduction of residual or reconnection gaps (black arrow). The two tags immediately adjacent to both side of the gap were subsequently termed "gap-related tags" (orange). All remaining tags from the first encirclement (pink line) were referred to as "non-gap tags" (red).

\section{Figure 2: Segments of Conduction Gaps Observed During the Index Procedure}

Black stars indicate segments with residual gaps after the first encirclement. White stars indicate segments with spontaneous reconnection gaps.

Abbreviations: $\mathrm{LRF}=$ left roof, LSPST $=$ left superior posterior, LIPST $=$ left inferior posterior, LINF $=$ left inferior, LIANT = left anterior, LSANT $=$ left superior anterior, LSPV = left superior pulmonary vein, $\mathrm{LIPV}=$ left inferior pulmonary vein, $\mathrm{RRF}=$ right roof, RSPST $=$ right superior posterior, $\mathrm{RIPST}=$ right inferior posterior, RINF = right inferior, RIANT $=$ right anterior, RSANT $=$ right superior anterior, RSPV $=$ right superior pulmonary vein, RIPV $=$ right inferior pulmonary vein, OTH $=$ other, $\mathrm{ESO}=$ esophagus.

\section{Figure 3: Summary of Acute Clinical Outcomes Associated with VS-Guided AF Ablation}

Abbreviations: VS = VISITAG SURPOINT, AF = atrial fibrillation, FPI $=$ first pass isolation, No Spont. $\mathrm{PVR}=$ No spontaneous pulmonary vein reconnection during the index procedure, $\mathrm{PVI}=$ pulmonary vein isolation.

\section{Figure 4: Comparison of VS Values and ITDs based on Gap Classifications}

VS values (A) and ITDs (B) based on residual gap classifications. (C) VS values and (D) ITDs based on reconnection gap classifications.

Abbreviations: VS $=$ VISITAG SURPOINT, ITD $=$ inter-tag distance.

Figure 5: Kaplan-Meier Curve for Recurrence of AT/AF after the Index Procedure

During median follow-up of 462 days $(\mathrm{N}=99)$, AT/AF recurrence was observed in $15(15.2 \%)$ patients.

Abbreviations: $\mathrm{AT}=$ atrial tachycardia, $\mathrm{AF}=$ atrial fibrillation, $\mathrm{CI}=$ confidence interval.

\section{Hosted file}

Surpoint Manuscript - for JCE Figure.pptx available at https://authorea.com/users/369030/ articles/487976-characterizing-clinical-outcomes-and-factors-associated-with-conductiongaps-in-visitag-surpoint-guided-catheter-ablation-for-atrial-fibrillation

\section{Hosted file}

Surpoint Manuscript - for JCE Table.pdf available at https://authorea.com/users/369030/ articles/487976-characterizing-clinical-outcomes-and-factors-associated-with-conductiongaps-in-visitag-surpoint-guided-catheter-ablation-for-atrial-fibrillation 